ISSN 1112-9867

\title{
LAMINATION METHOD OF FLOOD WADIS AND PROJECTION OF THE LAMINATED FLOOD HYDROGRAPH
}

\author{
M. Ladjel
}

Université Abderrahmane Mira, Bejaia, Algérie

Received: 27 June 2015 / Accepted: 23 December 2015 / Published online: 01 January 2016

\begin{abstract}
The catastrophic floods in semi-arid areas are often caused by floods storm that occur at any time during the year, including the hot season. The prevention of these floods could be done by the construction of small dam hills. This requires the control of theoretical concepts hydrological sizing, especially the hydrological structure to evacuate floods. We suggest a method to calculate the optimal regulation flow of the flood and also the development of a direct calculation formula of a laminated maximum flow. The analysis of the hydro graph's analogy at the input and the output of the dam, allow searching the dependencies between their characteristics. Knowing the characteristics of the hydrograph flood of the project and the reserved capacity for the amortization of the flood, we can directly determine the laminated maximum flow and project the hydrograph of the laminated flood.
\end{abstract}

Keywords: maximum flow; lamination; dam; flood; hydrograph; laminated flow.

Author Correspondence, e-mail: ladjel_mahmoud@yahoo.fr

doi: http://dx.doi.org/10.4314/jfas.v8i1.6

\section{INTRODUCTION}

Le climat semi-aride de l'Algérie impose la mobilisation des eaux de surface. Car tout développement implique une augmentation des besoins en eau des différents secteurs de l'économie. Le recours au stockage des ressources superficielles, dans des barrages, présente l'ultime solution avantageuse. Ces ouvrages coûteux exigent une protection contre les menaces des crues catastrophiques. Ces dernières peuvent inonder, en aval les terres agricoles, 
les zones industrielles et les agglomérations. Selon les données historiques récentes, relatives aux accidents des ouvrages hydrotechniques, le sous-dimensionnement des ouvrages d'évacuation est la cause de destruction de prés de $20 \%$ de tous les barrages dans le monde [4].

Le transit de la crue, dans le barrage, provoque un stockage d'eau temporaire, vu que les débits rentrants $Q_{i}$ sont, au départ, supérieurs aux débits sortants $q_{i}$. Ce phénomène est appelé régularisation de l'écoulement ou laminage des débits. Une fois le barrage plein jusqu'au niveau normal $N N$, le surplus des eaux de la crue pluviale est déversé progressivement en aval à travers l'évacuateur des crues, dont la capacité d'évacuation complète, correspond à la valeur du débit maximum laminé $q_{\max }$. Ainsi, le degré de laminage $r$ dépend du volume réservé au stockage temporaire $V_{s}$ et de la forme de l'hydrogramme de la crue. A cet effet, les calculs de laminage permettent le dimensionnement de déversoir des crues et le choix technico-économique adéquat.

\section{PRINCIPAUX OBJECTIFS DE LAMINAGE DES CRUES DES OUEDS}

Le principal objectif de laminage est d'empêcher les inondations en bief aval, par le laminage des débits sortants du barrage. Que le plus grand débit, sortant du barrage, ne doit pas dépasser la capacité d'évacuation du lit en zone aval.

Les tâches essentielles des calculs de laminage des débits maxima des crues sont [8]:

1) Déterminer le débit maximum laminé et le niveau maximum d'eau dans le barrage (PHE).

2) Déterminer la largeur du déversoir, correspondant au débit maximum laminé $q_{p \%}$ donné.

3) Déterminer le volume de stockage temporaire $V_{s}$ dans le barrage.

\section{CHOIX DE L'HYDROGRAMME DE PROJET}

Le choix de l'hydrogramme de la crue de projet est nécessaire, pour le dimensionnement des ouvrages d'évacuation et pour leur estimation économique. Il doit faire l'objet d'une analyse hydrologique appropriée. Pour les bassins versants jaugés, on adopte un hydrogramme relatif moyen, parmi tous les hydrogrammes des crues rares, dont les débits maxima et les volumes d'écoulement sont proches de celle de la crue de projet [9]. Pour les bassins non jaugés, surtout les petits, le choix se fait sur la base d'un modèle régional de crue [2, 10]. 


\section{DETERMINATION DU DÉBIT MAXIMUM LAMINÉ}

Sachant qu'on peut exprimer le débit maximum d'une crue, comme suit $[1,5,9]$.

$$
Q_{p \%}=0.28 k \frac{V_{c}}{T}
$$

où:

0.28 - coefficient, qui tient compte des unités physiques de $Q_{P \%}$, de $V_{c}$ et de $T ; k$ coefficient de forme de la crue, est égal au rapport de débit maximum $Q_{p \%}$ sur le débit moyen $\bar{Q} ; T$ - temps de base de la crue.

De la même façon, le débit maximum laminé est exprimé par [6, 7] :

$$
q_{p \%}=0.28 k^{\prime} \frac{V_{c}-V_{s}}{T}
$$

où:

$k^{\prime}$ - coefficient de forme de l'écoulement non régularisé; $V_{s}=V(N M)-V(N N)$ est le volume de stockage, compris entre le niveau maximum $N M$ et le niveau normal $N N$ (fig.1).

En divisant (2) par (1), on obtient la relation suivante:

$$
q_{p \%}=\frac{k^{\prime}}{k} \frac{V_{c}-V_{s}}{V_{c}} Q_{p \%}
$$

Dans la relation (3), toutes les variables sont connues, hormis le coefficient $k^{\prime}$, qu'il faut identifier. En admettant une relation linéaire entre le coefficient $k^{\prime}$ et le rapport $q_{p \%} / Q_{p \%}$, soit $[6,7]$.

$$
k^{\prime}=a \frac{q_{p \%}}{Q_{p \%}}+c
$$

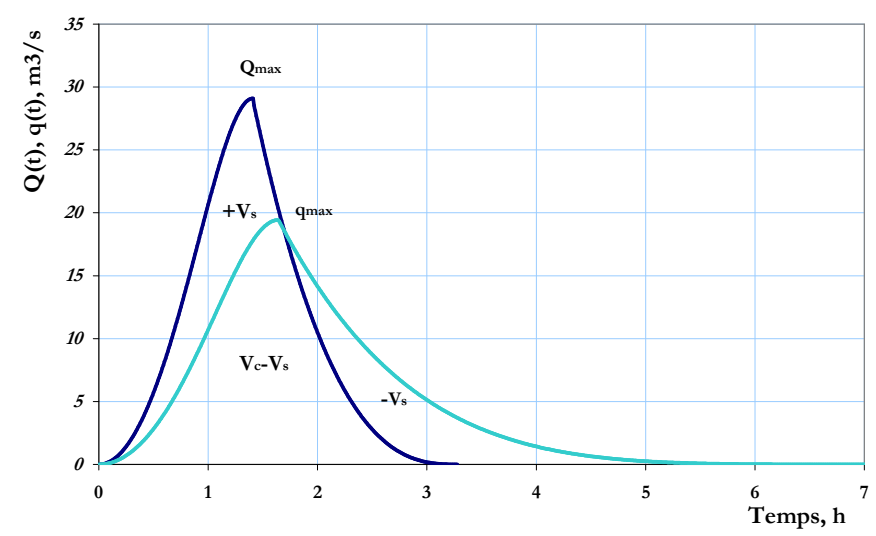

Fig.1. Différentes caractéristiques de l'hydrogramme 
et en remplaçant l'expression (4) dans (3), après quelques transformations, on obtient :

$$
q_{p \%}=\frac{c}{k \frac{V_{c}}{V_{c}-V_{s}}-(k-c)} Q_{p \%}
$$

avec

$$
r=\frac{c}{k \frac{V_{c}}{V_{c}-V_{s}}-(k-c)}
$$

Sachant que la forme triangulaire de l'hydrogramme de la crue correspond aux valeurs limites $k=2$ et $r=\frac{V_{c}-V_{s}}{V_{c}}$ [3]. Ainsi, la vérification du coefficient $c$ peut se faire par l'égalité suivante [7]:

$$
\frac{V_{c}-V_{s}}{V_{c}}=\frac{c}{k \frac{V_{c}}{V_{c}-V_{s}}-(k-c)}
$$

D'où, l'on tire la valeur de $c=2$, correspondant à la valeur minimale du coefficient de forme $k_{\text {min }}$.

Enfin, le coefficient de laminage des débits maxima s'exprime par la relation:

$$
r=\frac{k_{\min }}{k \frac{V_{c}}{V_{c}-V_{s}}-\left(k-k_{\min }\right)}
$$

avec: $k$ - coefficient de forme de la crue de projet, égal au rapport $Q_{\max } / Q_{\text {moyen }} ; k_{\min }$ - valeur minimale théorique du coefficient de forme de la crue, égale à 2 pour un hydrogramme de forme triangulaire parfaite [3]; $V_{c}$ - volume de la crue en $m^{3}$ et $V_{s}$ - volume de stockage en $m^{3}$.

\section{TENIR COMPTE DU NIVEAU DYNAMIQUE DU PLAN D'EAU}

Les calculs du laminage, en considérant le volume statique avec un plan d'eau horizontal, provoque une augmentation du niveau d'eau $h$ sur le seuil déversant et provoque une surestimation des débits déversés $q(h)$ calculés, d'où une sous-estimation de la largeur de l'évacuateur de crues [8]. Pour tenir compte du volume dynamique d'eau dans le barrage, on doit chercher d'une correction $\lambda$, qui doit être inférieure à l'unité, pour passer de la valeur du 
niveau statique $h_{s}$ au niveau dynamique $h_{d}$, par la relation $h_{d}=\lambda h_{s}$. La forme dynamique du plan d'eau, durant le transit de la crue par le barrage, dépend des caractéristiques morphomètriques de la cuvette du barrage, qui influent sur les caractéristiques hydrauliques de stockage temporaire des eaux dans le barrage. A cet effet, la correction $\lambda$ peut être exprimée, en fonction des seules caractéristiques morphomètriques de la cuvette pleine, comme suit:

$$
\lambda=\frac{1}{1+\left(\frac{B}{L}\right)^{H / H_{m}}}
$$

où:

$B$ - largeur moyenne du plan d'eau en $m$,

$L$ - longueur du plan d'eau, suivant le tracé du cours d'eau en $m$,

$H$ - niveau maximum de l'eau, en bief amont en $m$,

$H_{m}$ - profondeur moyenne d'eau, dans le barrage en $m$.

La correction $\lambda$ a été estimée pour quelques sites de retenues collinaires, situées à l'Est de l'Algérie. Cette correction montre que plus le rapport $H_{m} / H$ est petit, le niveau dynamique $h_{d}$ s'approche du niveau statique $h_{s}$ (fig.2).

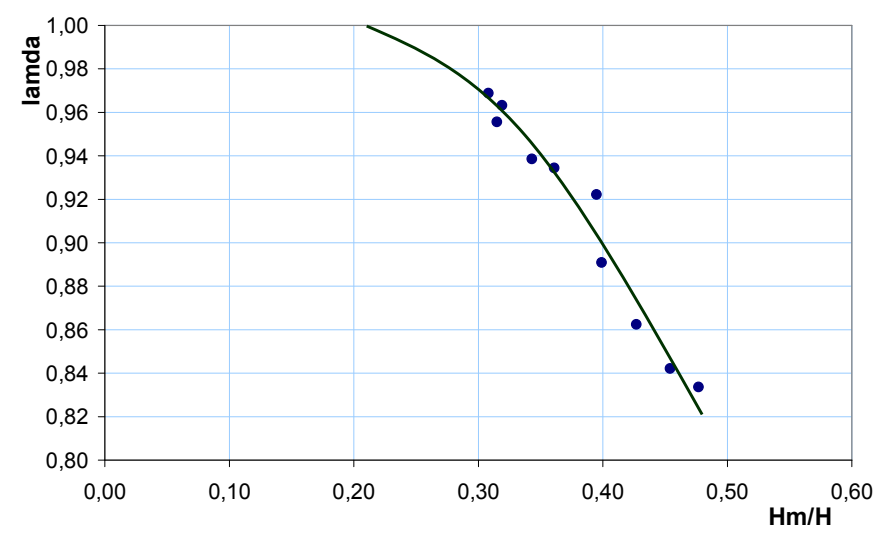

Figure 2 : Graphique de la dépendance $\lambda=f\left(B, L, H_{m}, H\right)$

\section{PROJECTION DE L'HYDROGRAMME LAMINÉ}

Les principales caractéristiques des hydrogrammes, de la crue de projet et de la crue laminée, sont respectivement les débits $Q_{p \%}$ et $q_{p \%}$, les volumes $V_{c}$ et $V_{s}$ et les temps de base $T$ et 
$T^{\prime}$. Le temps de base, de la crue laminée, est lié au temps de base de la crue de projet par la relation suivante $[6,7]$ :

$$
T^{\prime}=\frac{T}{r^{2}}
$$

Aussi, le temps de monté de l'hydrogramme laminé $t_{m}^{\prime}$ est lié avec le temps de montée de la crue de projet, par:

$$
t_{m}^{\prime}=\left[\gamma\left(1-r^{\frac{1}{m}}\right)+1\right] t_{m}
$$

avec $\gamma=\frac{T}{t_{m}}-1$ et $m$ coefficient de forme de la crue.

Sachant que $T^{\prime}=\left(\gamma^{\prime}+1\right) \cdot t_{m}^{\prime}$ et en tenant compte de la relation (10), on obtient:

$$
\gamma^{\prime}=\frac{\gamma+1}{r^{2}\left[\gamma \cdot\left(1-r^{\frac{1}{m}}\right)+1\right]}-1
$$

La construction de l'hydrogramme de la crue laminée est possible, quand à tout instant $t_{k}$, on connait le débit courant $Q_{k}$ de la crue de projet.

Pour la phase de la montée, à l'instant $t_{i}$, on tire de l'hydrogramme de la crue le débit correspondant $Q_{i}$ et on calcule les coordonnées:

$$
t_{i}^{\prime}=\frac{t_{m}^{\prime}}{t_{m}} t_{i}
$$

et

$$
q_{t_{i}^{\prime}}=r Q_{t_{i}}
$$

Pour la phase de la décrue, à l'instant $t_{j}$ on tire de l'hydrogramme de la crue le débit correspondant $Q_{j}$ et on calcule les coordonnées:

$$
t_{j}^{\prime}=\frac{t_{d}^{\prime}}{t_{d}} t_{j}
$$

et

$$
q_{t_{i}^{\prime}}=r Q_{t_{i}}
$$


Ainsi toutes les caractéristiques fondamentales de l'hydrogramme de la crue laminée $q_{p \%}$, $t^{\prime}{ }_{m}, t^{\prime}{ }_{d}=\gamma^{\prime} t^{\prime}{ }_{m}$ et $T^{\prime}=\left(\gamma^{\prime}+1\right) t_{m}^{\prime}$ sont exprimées directement à travers celle de l'hydrogramme de la crue, déjà connu.

\section{CONCLUSION}

La mobilisation des ressources en eau superficielle, dans des retenues et barrages, est inévitable dans les pays de climat semi-aride et aride. Ces ouvrages coûteux pour les pays pauvres, doivent être sécurisés contre les conséquences des grandes crues.

La maîtrise des calculs de laminage permet le dimensionnement des ouvrages d'évacuation des crues catastrophiques. Le choix de l'hydrogramme de la crue de projet doit faire l'objet d'une analyse hydrologique appropriée.

Le coefficient de laminage dépend essentiellement du volume de la crue, du volume de stockage temporaire et aussi de la forme de la crue.

La ressemblance de l'hydrogramme de la crue de projet et l'hydrogramme laminé permet d'identification des caractéristiques du temps de ce dernier qui permettent de le projeter.

\section{RÉFÉRENCES}

[1] Бефани А. Н., 1989. вопросы региональной гидрологии. Паводочный сток (ливневые наводнений). Киев, 130 pp.

[2] Волчек А.А., Шелест Т.А., 2013. Моделирование гидрографов дождевых паводков рек Беларуси при отсутсвии данных гидрометрических наблюдений, Вестник Брестского государственного технического университета. №2.

[3] Кочерин Д. И., 1927. Определение отверстий искуственных сооружений с учетом хода паводочного притока и наполнения пруда, Тр. Моск. Ин-та инженеров транспорта, вып. VI.

[4] Крицкий С. Н. , Менкель М. Ф., 1981. Гидродогические основы управления речным стоком. Издатеьство -НАУКА- Москва, 255 рр.

[5] Ladjel M., 1995. Crue pluviales - Débit maximum laminé. $9^{\text {ème }}$ Conférence régionale Afro-Asiatique des irrigations et du drainage. Alger.

[6] Ladjel M., 1999. Construction de l'hydrogramme de la crue laminée par un barrage. Colloque Maghrébin de Génie Civil, CMGC'99. Annaba. 
[7] Ladjel M., 2004. Projection de l'hydrogramme de la crue de projet et de l'hydrogramme laminé. Premier Séminaire National sur les Sciences de la Terre au Service du Développement Durable, Tébessa.

[8] Плешков Я. Ф., 1975. Регулирование речного стока. Гидрометеоиздат, Л., 552 рр.

[9] Соколовский Д. Л., 1969. Речной сток. Гирометеоиздат, Ленинград. 539 pp.

[10] Petits Barrages, 2002. Recommandations pour la conception, la réalisation et le suivi. Edition 'Cemagref, 173 pp. 


\section{METHODE DE LAMINAGE DES CRUES PLUVIALES DES OUEDS \\ ET PROJECTION DE L'HYDROGRAMME DE LA CRUE LAMINEE}

\section{RESUME}

Les inondations catastrophiques, en zones semi-arides, sont souvent provoquées par les crues pluviales, qui peuvent se manifester, même durant l'été. Lutter contre ces inondations peut se faire par la construction des retenues collinaires. Ceci exige la maîtrise des concepts théoriques de dimensionnement hydrologique, surtout les ouvrages d'évacuation des crues. On propose une méthode de calcul de régularisation optimale des débits des crues et une formule de calcul du débit maximum laminé. L'analyse de l'analogie des hydrogrammes, à l'entrée et à la sortie du barrage, a permis d'établir des dépendances analytiques entre leurs caractéristiques. Connaissant les caractéristiques de l'hydrogramme de la crue de projet et la capacité réservée pour l'amortissement de la crue, on peut déterminer directement le débit maximum laminé et projeter l'hydrogramme de la crue laminée.

Mots clé: débit maximum; laminage; barrage; déversoir; crue ; hydrogramme; débit laminé.

\section{How to cite this article:}

Ladjel M. Lamination method of flood wadis and projection of the laminated flood hydrograph. J. Fundam. Appl. Sci., 2016, 8(1), 83-91. 